\title{
Towards a Formal Semantics for a Structurally Dynamic Noncausal Modelling Language
}

\author{
John Capper \\ University of Nottingham \\ Nottingham, UK \\ jjc@cs.nott.ac.uk
}

\author{
Henrik Nilsson \\ University of Nottingham \\ Nottingham, UK \\ nhn@cs.nott.ac.uk
}

\begin{abstract}
Modelling and simulation languages are evolving rapidly to support modelling of systems of ever increasing size and complexity. A relatively recent development in the area of physical modelling is the noncausal modelling languages. They support a declarative, highly modular modelling approach, promoting the reuse of components. Modelica is a prime example of this class of languages. However, the mainstream representatives of this class of languages provide limited support for higher-order modelling and structurally dynamic systems. Moreover, the semantics of this class of languages remains a relatively unexplored area. Functional Hybrid Modelling (FHM) is a novel approach to noncausal, hybrid modelling that aims to address these concerns. In this paper, we give a semantics to the discrete part of a simple FHM language expressed in dependent type theory. We use Normalisation by Evaluation to produce a type-preserving and terminating normalisation procedure, the latter property being particularly important for FHM as highly structurally dynamic systems are supported by computing new system configurations during simulation. Furthermore, our implementation has been carefully structured to allow continuous aspects of the semantics to be described separately, in whatever way is deemed appropriate, while retaining the ability to describe precisely how a system evolves in response to discrete events.
\end{abstract}

Categories and Subject Descriptors D.3.2 [Programming Languages]: Language Classifications-applicative (functional) languages, specialized application languages; D.3.1 [Programming Languages]: Formal Definitions and Theory-Semantics

General Terms Design, Languages, Theory, Verification

Keywords Noncausal Hybrid Modelling, Normalisation, Dependent Types, Formal Verification

\section{Introduction}

Digital computers have been used for modelling and simulation since their inception. The development of computers with ever more performance has made it feasible to simulate systems of rapidly increasing size and complexity. This in turn has spurred the creation of dedicated languages designed to facilitate modelling

Permission to make digital or hard copies of all or part of this work for personal or classroom use is granted without fee provided that copies are not made or distributed for profit or commercial advantage and that copies bear this notice and the full citation on the first page. To copy otherwise, to republish, to post on servers or to redistribute to lists, requires prior specific permission and/or a fee.

TLDI'12, January 28, 2012, Philadelphia, PA, USA.

Copyright (C) 2012 ACM 978-1-4503-1120-5/12/01 . .\$10.00 and simulation for a range of more or less specific application domains. Noncausal (or acausal) modelling languages are a relatively recent development in the area of physical modelling. They support a declarative, equation-based, highly modular approach for modelling dynamical systems, promoting the reuse of components [8]. Partly because of the focus on components, and partly because of design influences from object-oriented programming languages, many of these languages are referred to as object-oriented modelling languages. Modelica [23] is one prominent example.

However, the mainstream representatives of the noncausal modelling languages provide limited support for higher-order modelling and hybrid systems, in particular structural dynamism $[4,15$, 31]. Hybrid refers to systems having both continuous and discrete components [18], which in modelling terms translates into continuous variables evolving in continuous time, combined with discrete changes of variables or system structure. An additional concern is that the hybrid support is typically realised by adding imperative constructs to the language where a declarative perspective would be more desirable. It is also of note that the semantics of this class of languages remains a relatively unexplored area.

Functional Hybrid Modelling (FHM) is a novel approach to noncausal hybrid modelling that aims to address these concerns [26]. FHM combines a functional language with first-class abstractions that capture the essence of noncausal and hybrid modelling, yielding a stratified language with two levels: a functional level and a modelling level. This design keeps the resulting modelling language simple and facilitates semantic investigation. Yet the approach is very expressive: in particular, highly structurally dynamic systems are supported; i.e., systems where the number of structural configurations is too large for an explicit enumeration to be practical or possible. FHM achieves this by allowing new structural configurations to be computed as needed, during simulation.

This paper seeks to put the semantics of FHM languages and, more broadly, modelling languages supporting higher-order modelling and structural dynamics on a firm foundation. To that end, we express the semantics in dependent type theory using Normalisation by Evaluation (NbE) [1]. This allows us to prove key correctness properties. Additionally, we hope the semantics will be useful as a basis for further investigations, for example, into domainspecific type-systems for this class of languages [6, 25], as well as a reference for implementers and users. Our general approach may also be of interest in the context of other two- or multi-level languages. Many embedded domain-specific languages [19] fall into this category. One example is Functional Reactive Programming (FRP) $[13,29]$, which was a source of inspiration for FHM.

We focus on the discrete, as opposed to continuous aspects of the FHM semantics in this paper; i.e., how a modularly composed model is transformed into a "flat" system of equations that describe the continuous-time dynamic behaviour for a particular structural 
configuration, and how, conversely, new structural configurations are computed in response to events triggered by this behaviour. We do not concern ourselves with the continuous part of the semantics, beyond that it is (an approximation of) the solution to the equations describing a structural configuration, if a solution exists. This separation of concerns is deliberate: how prescriptive the continuous aspect of the semantics should be depends on the purpose at hand; consequently, we wish to formalise the discrete part of the semantics in such a way that it fits with any reasonable approach to describing the continuous part. ${ }^{1}$ For example, one option is to view our semantics as describing the computation of fragments of a possibly infinite hybrid automaton [18] on demand (see Sect. 6).

In more detail, our specific contributions are:

- We give a semantics for the discrete part of a simple FHM language expressed in dependent type theory using $\mathrm{NbE}$. We use Agda as our metalanguage. The normalisation procedure produces canonical normal forms, is type preserving, and is both total and terminating. Termination is of particular relevance for FHM as new system configurations are computed at discrete points in time, during simulation.

- We give a coinductive formulation of dynamism and the generation of new structural configurations, constituting a proof that the generation process is productive. This is of particular importance for highly structurally dynamic systems, and to the authors' knowledge, is the first such result in this setting.

- We provide a semantics that is carefully structured so as to allow the continuous aspects to be described separately, in whatever way is most appropriate for the purpose at hand, while retaining the ability to describe precisely how a system evolves in response to discrete events.

The rest of the paper is structured as follows. The next section gives a brief introduction to FHM. Section 3 defines the precise language of this paper. Section 4 then discusses the normalisation, followed by a discussion on structural dynamism in Sect. 5. We consider related work in Sect. 6 and finish with notes on future work and give conclusions in Sect. 7.

\section{Functional Hybrid Modelling}

\subsection{Fundamentals}

Modelling the dynamic aspects (behaviour over time) of a physical system mathematically often requires the use of (temporal) differential equations. If algebraic equations are used as well, and if equations can be implicit, the result is known as an (implicit) Differential Algebraic system of Equations, or DAE for short. A DAE constitutes a noncausal model, as implicit equations are inherently undirected (no syntactic requirement restricting the left- or righthand side to a single variable). It turns out that such a system can be expressed modularly, by composing DAE fragments that model individual physical system components [8], and that such DAE fragments are highly reusable as there are no a priori assumptions about which specific variable is going to be used to solve for each equation [9, pp. 1-19, 253-396].

The idea of Functional Hybrid Modelling (FHM) is to enrich a functional language with key notions for noncausal modelling, thus creating a stratified language with two levels: a functional level dealing with time-invariant entities, and a modelling or signal level concerned with time-varying entities, or signals. Facilities for mediating between the two levels are also provided. A concrete example of an FHM language is Hydra [26], which has been

\footnotetext{
${ }^{1}$ This approach mirrors the use of a broad spectrum of (numerical) solvers in current state-of-the-art, industrial-strength (hybrid) modelling languages: which solver(s) to use depends on the nature of the problem.
}

implemented as a domain-specific language embedded in Haskell $[15,16]$. The central FHM abstraction is the signal relation. A signal relation is an encapsulated (fragment of a) DAE: it has an interface, consisting of a number of interface variables, and a set of equations expressing relations between the interface variables and any additional local variables. The name 'signal relation' reflects our concern with temporal differential equations, which implies that the variables at this level in general stand for signals. We refer to such variables as signal variables, in contrast to $\lambda$-bound, functional-level variables that stand for time-invariant entities.

Object-oriented modelling languages, like Modelica, provide a number of mechanisms for reuse and composition of equation fragments, including inheritance. In contrast, FHM provides a single mechanism, signal relation application, that instantiates the contained equations. Furthermore, signal relations are first-class entities at the functional level in FHM, meaning they can be manipulated programmatically just like any other type of value. This is sometimes referred to as higher-order modelling ${ }^{2}$ [4] and amounts to (simple) metamodelling, functionality that otherwise would have to be provided through a separate metamodelling language [27].

Moreover, with just a minimum of additional language constructs, higher-order modelling allows highly structurally dynamic systems to be expressed: all that is needed are the means to compute and integrate new model fragments into a model during simulation, at discrete points in time, called events, not just before simulation starts. This is what makes an FHM language hybrid: encompassing both continuous and discrete notions. Indeed, the ease with which higher-order modelling can express structural dynamics was partly what motivated the research into FHM in the first place [26].

\subsection{A Simple Electrical Circuit in Hydra}

To illustrate the basic ideas of FHM, we model the electrical circuit in Fig. 1(a) in Hydra. An understanding of the underlying physics is not central to this paper; only the way the topology of the circuit is expressed through components and connections is important. Our initial example has a static structure; we return to structural dynamism in the next subsection. As Hydra is work in progress, and to prevent minor syntactic details from getting in the way of the presentation, we adopt a somewhat idealised Hydra syntax.

We start by modelling a generic electrical component with two pins. It captures aspects common to all such components:

$$
\begin{aligned}
& \text { twoPin }:: S R(\text { Pin, Pin, Voltage }) \\
& \text { twoPin }=\text { sigrel }(p, n, u) \text { where } \\
& p . v-n . v=u \\
& p . i+n . i=0
\end{aligned}
$$

$S R$ is the type constructor for signal relations. The signal relation two Pin is notionally a relation between three signals. However, the type Pin is a record type describing an electrical connection. It has fields $v$ for voltage and $i$ for current. As a signal of products is isomorphic to a product of signals, the signal relation twoPin can be seen as a relation among five physical quantities: the potential $p . v$ at the positive pin $p$, the current $p . i$ into the positive pin $p$, the potential $n . v$ at the negative pin $n$, the current $n . i$ into the negative pin $n$, and the voltage $u$ across the component.

Specific electrical two-pin components can now be defined as extensions of the twoPin model using signal relation application, denoted by $\diamond$. The idea is simple: the equations describing the applied signal relation are copied into the context of the application, substituting the expressions the relation was applied to for its formal arguments and renaming local variables as needed to avoid

\footnotetext{
${ }^{2}$ As it is reminiscent of higher-order functions. Not to be confused with other meanings of higher-order, such as higher-order derivatives.
} 


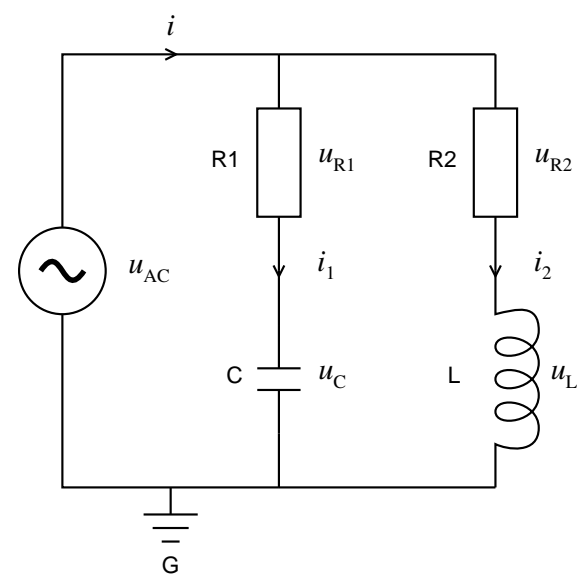

(a) Circuit

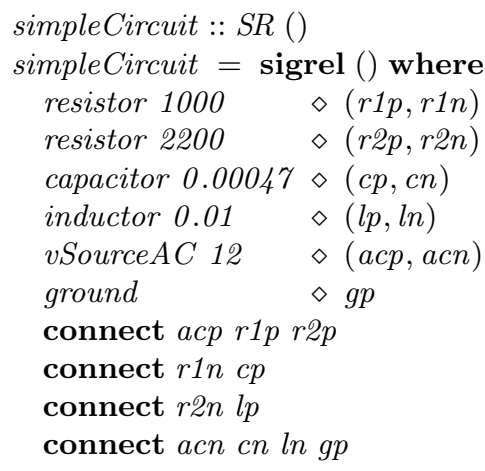

(b) Hydra model

Figure 1: A simple electrical circuit and its model in Hydra.

name clashes. Additional equations for describing componentspecific behaviour are then added. For example, a capacitor model:

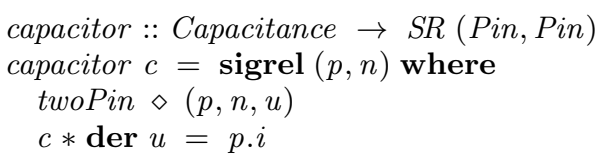

Here, $u$ is a local variable. For the purpose of this paper, there is no need to explicitly declare local variables. The function der denotes the derivative with respect to time of the supplied signal.

The capacitor model is parametrised by modelling it as a function from capacitance to a signal relation, thus exploiting the first-class status of signal relations. Because the parameter $c$ is a functional-level argument, not a variable denoting a signal (like $u$ or $p . i$ ), its value will remain unchanged throughout the lifetime of the returned relation. Signal relations can even be parametrised on other signal relations in the same way.

To illustrate signal relation application, let us expand

capacitor $0.00047 \diamond(c p, c n)$

using the definitions of twoPin and capacitor. Note that capacitor 0.00047 to the left of the signal relation application operator $\diamond$ is a functional-level expression that will evaluate to a signal relation that in turn will be applied to $(c p, c n)$ at the signal level. The following are the three resulting equations, where $u^{\prime}$ is a fresh name (in lieu of $u$ ) to avoid any name clashes (e.g. due to any additional instantiations of capacitor):

$$
\begin{array}{ll}
c p . v-c n . v & =u^{\prime} \\
c p . i+c n . i & =0 \\
0.00047 * \operatorname{der} u^{\prime} & =c p . i
\end{array}
$$

Figure 1(b) shows a complete Hydra model for our circuit. A Modelica-inspired connect-construct provides a convenient abbreviation for connection equations. This is just syntactic sugar that is expanded to basic equations: equality constraints for connected potential quantities (here voltage) and a sum-to-zero equation for connected flow quantities (here current) [17]. For example:

connect $a c p \quad r 1 p r 2 p$

gets expanded into:

$$
\begin{aligned}
& a c p . i+r 1 p \cdot i+r 2 p \cdot i=0 \\
& a c p . v=r 1 p \cdot v \\
& r 1 p \cdot v=r 2 p \cdot v
\end{aligned}
$$

We assume models of other standard electrical components. As there are no inputs or outputs, the model becomes a nullary relation.

\subsection{Dynamic Structure}

To express structurally dynamic systems, Hydra employs a switch construct that allows equations to be brought into and removed from a model as needed:

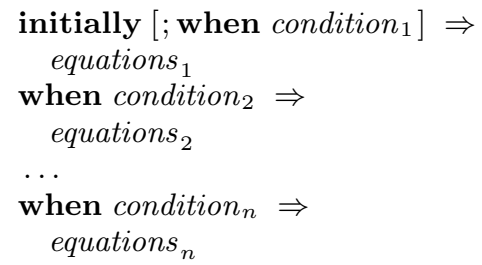

Only the equations from one branch are active at any one point in time. The equations of a branch are switched in whenever the condition guarding the branch becomes true, at which point those from the previously active branch are switched out. The keyword initially designates the initially active branch. An optional condition allows for the initial branch to be re-activated later. Should more than one switch condition within a switch construct trigger simultaneously, the branches are prioritised by syntactic ordering.

Whenever a switch occurs, continuous simulation stops, a new system of equations is generated, turned into simulation code, and simulation resumes. As simulation amounts to solving an initial value problem, i.e., finding out how the system evolves over time from a given initial state, the new system of equations must be properly initialised. Finding a consistent initial state is a hard problem in general. Here, this problem must be solved after each structural reconfiguration, as state variables may then change discontinuously. Indeed, entirely new state variables may be introduced. Thus, Hydra does not attempt to provide any automatic solution. Instead, explicit initialisation and reinitialisation equations, active only at the start and after a structural reconfiguration, respectively, allow the modeller to express modelling intent such as continuity assumptions (e.g., the charge of a capacitor must not change abruptly).

\section{A Simple FHM Language}

In this section we introduce the language $\mu-\mathrm{H}$. It is closely related to Hydra (Sect. 2) but has been simplified to facilitate formalisation and metatheoretical study, retaining only features of interest to this paper. In particular, the functional host language is the simply typed $\lambda$-calculus as opposed to Haskell.

\subsection{Syntax}

Like Hydra, $\mu-\mathrm{H}$ is stratified into two levels; see Fig. 2. The category $t$ specifies the syntax of the functional host language: essen- 


\begin{tabular}{|c|c|}
\hline & functional-level term \\
\hline$x$ & variab \\
\hline lit & numeric liter \\
\hline$t_{1} t_{2}$ & applicati \\
\hline$\lambda x . t$ & abstracti \\
\hline sigrel $z$ where $q$ & signal relati \\
\hline$s::=$ & signal-level ter \\
\hline$z$ & variak \\
\hline$t$ & functional-level te \\
\hline unit & \\
\hline $\operatorname{der} s$ & time derivati \\
\hline$s_{1}+s_{2}$ & additi \\
\hline$s_{1}-s_{2}$ & subtracti \\
\hline$s_{1} * s_{2}$ & multiplicati \\
\hline$\pi_{1} s$ & first projecti \\
\hline$\pi_{2} s$ & second projecti \\
\hline$\left(s_{1}, s_{2}\right)$ & \\
\hline
\end{tabular}

Figure 2: Functional- and signal-level term syntax.

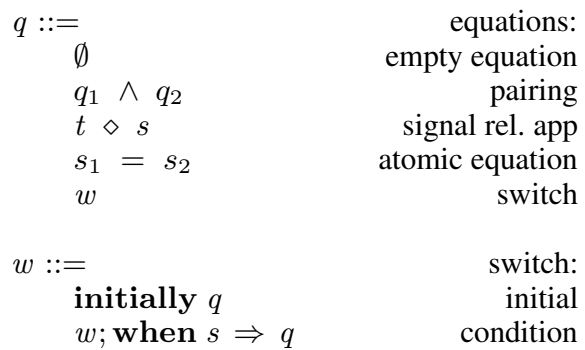

Figure 3: Equation and switch syntax.

tially the simply typed $\lambda$-calculus extended with signal relations. The category $s$ describes signal-level expressions. With the exception of products, the details of signal-level expressions are of little concern in this paper as we only wish to flatten the modular system of equations, not perform any other symbolic transformations on them. We retain some additional structure to get the basic intuitions across. For simplicity, we use an uninterpreted numeric type: the reader can consider it a placeholder for a more appropriate type, such as Double. The categories $x$ and $z$ are $\lambda$-variables and signal variables, respectively.

Figure 3 gives the syntax of systems of equations, category $q$, including switch blocks, category $w$. The equations form a commutative monoid $\langle q, \wedge, \emptyset\rangle$ (order and grouping is irrelevant) and we will exploit the associativity and unit laws throughout the remainder of this paper. We take the switch condition to simply be a signal-level term. The condition triggers a switch whenever the denoted signal crosses zero. This suffices for our purposes, and reflects how current industrial-strength numerical solvers work.

\subsection{Typing}

Our language has a simple type system that is stratified into two levels to reflect the term syntax: see Fig. 4. The functional level consists of function spaces and signal relation types. Signal relation types are parametrised by a signal-level type, which consists of the unit type (for representing nullary signal relations) and signal-level products. The numeric type Num appears at both levels.

The type system is specified by four relations, one for each syntactic category given in Sect. 3.1: see Fig. 5 and Fig. $6 . \Gamma$ and $\Delta$ are the functional-level and signal-level typing contexts, respectively. $\epsilon$ denotes the empty context. An individual equation

$$
\begin{aligned}
& \tau::= \\
& \text { Num } \\
& \tau_{1} \rightarrow \tau_{2} \\
& S R \sigma \\
& \sigma::= \\
& \text { Unit } \\
& \text { Num } \\
& \sigma_{1} \times \sigma_{2}
\end{aligned}
$$

Figure 4: Types.

$$
\begin{aligned}
& \frac{x: \tau \in \Gamma}{\Gamma \vdash x: \tau} \quad(\mathrm{T}-\mathrm{VAR}) \quad \overline{\Gamma \vdash \text { lit }: N u m} \\
& \frac{\Gamma, x: \tau_{1} \vdash t: \tau_{2}}{\Gamma \vdash \lambda x . t: \tau_{1} \rightarrow \tau_{2}}
\end{aligned}
$$

$$
\begin{gathered}
\frac{\Gamma \vdash t_{1}: \tau_{2} \rightarrow \tau_{1} \quad \Gamma \vdash t_{2}: \tau_{2}}{\Gamma \vdash t_{1} t_{2}: \tau_{1}} \\
\frac{\Gamma ; \epsilon, z: \sigma \vdash_{q} q}{\Gamma \vdash \text { sigrel } z \text { where } q: S R \sigma}
\end{gathered}
$$

(a) The functional-level typing relation $\Gamma \vdash t: \tau$.

$$
\begin{array}{cc}
\frac{z: \sigma \in \Delta}{\Gamma ; \Delta \vdash_{s} z: \sigma} & \text { (T-SVAR) } \quad \frac{z: \sigma \notin \Delta}{\Gamma ; \Delta \vdash_{s} z: N u m} \\
& \frac{\Gamma \vdash t: N u m}{\Gamma ; \Delta \vdash_{s} t: N u m} \quad \text { (T-FUN) }
\end{array}
$$

$$
\begin{gathered}
\frac{\Gamma ; \Delta \vdash_{s} \text { unit }: \text { Unit }}{\Gamma ; \Delta \vdash_{s} s: N u m} \quad \text { (T-UNIT) } \\
\frac{\Gamma ; \Delta \vdash_{s} \operatorname{der} s: N u m}{\otimes \in\{+,-, *\}} \text { (T-DER) } \\
\frac{\Gamma ; \Delta \vdash_{s} s_{1}: N u m \quad \Gamma ; \Delta \vdash_{s} s_{2}: N u m}{\Gamma ; \Delta \vdash_{s} s_{1} \otimes s_{2}: N u m} \\
\frac{\Gamma ; \Delta \vdash_{s} s_{1}: \sigma_{1} \quad \Gamma ; \Delta \vdash_{s} s_{2}: \sigma_{2}}{\Gamma ; \Delta \vdash_{s}\left(s_{1}, s_{2}\right): \sigma_{1} \times \sigma_{2}} \quad \text { (T-PR- } \\
\frac{\Gamma ; \Delta \vdash_{s} s: \sigma_{1} \times \sigma_{2}}{\Gamma ; \Delta \vdash_{s} \pi_{i} s: \sigma_{i}} \quad i \in\{1,2\} \quad \text { (T-PROJ) }
\end{gathered}
$$

(b) The signal-level typing relation $\Gamma ; \Delta \vdash_{s} s: \sigma$.

Figure 5: Functional- and signal-level typing relations.

has no type, but for an equation to be well-typed, its components need to be typed consistently. A system of equations is well-typed if its constituent equations are also well-typed.

Hydra revisited. At this point, it is helpful to revisit the examples given in the previous section to see how $\mu-\mathrm{H}$ is related to Hydra. Below, the twoPin and capacitor examples have been translated into core syntax (we retain Haskell-style type signatures to clarify 


$$
\begin{gathered}
\frac{\Gamma ; \Delta \vdash_{q} \emptyset}{(\text { T-EMPTY })} \\
\frac{\Gamma ; \Delta \vdash_{q} q_{1} \quad \Gamma ; \Delta \vdash_{q} q_{2}}{\Gamma ; \Delta \vdash_{q} q_{1} \wedge q_{2}} \quad(\mathrm{~T}-\mathrm{PA} \\
\frac{\Gamma \vdash t: S R \sigma \quad \Gamma ; \Delta \vdash_{s} s: \sigma}{\Gamma ; \Delta \vdash_{q} t \diamond s} \quad(\mathrm{~T}-\mathrm{S} / \sigma \\
\frac{\Gamma ; \Delta \vdash_{s} s_{1}: \sigma \quad \Gamma ; \Delta \vdash_{s} s_{2}: \sigma}{\Gamma ; \Delta \vdash_{q} s_{1}=s_{2}} \\
\frac{\Gamma ; \Delta \vdash_{w} w}{\Gamma ; \Delta \vdash_{q} w} \quad(\mathrm{~T}-\mathrm{S} \text { witCH })
\end{gathered}
$$

(a) The equation typing relation $\Gamma ; \Delta \vdash_{q} q$.

$$
\begin{gathered}
\frac{\Gamma ; \Delta \vdash_{q} q}{\Gamma ; \Delta \vdash_{w} \text { initially } q} \quad \text { (T-Initially) } \\
\frac{\Gamma ; \Delta \vdash_{w} w \quad \Gamma ; \Delta \vdash_{s} s: N u m \quad \Gamma ; \Delta \vdash_{q} q}{\Gamma ; \Delta \vdash_{w} w ; \text { when } s \Rightarrow q} \quad(\text { T-WH }
\end{gathered}
$$

(b) The switch typing relation $\Gamma ; \Delta \vdash_{w} w$.

Figure 6: Equation and switch typing relations.

the types and allow ourselves to use named definitions as an abbreviation):

$$
\begin{aligned}
& \text { Pin }=\text { Num } \times \text { Num } \\
& \text { twoPin }:: \text { SR }((\text { Pin } \times \text { Pin }) \times \text { Num }) \\
& \text { twoPin = sigrel } z \text { where } \\
& \quad \pi_{1}\left(\pi_{1}\left(\pi_{1} z\right)\right)-\pi_{1}\left(\pi_{2}\left(\pi_{1} z\right)\right)=\pi_{2} z \wedge \\
& \pi_{2}\left(\pi_{1}\left(\pi_{1} z\right)\right)+\pi_{2}\left(\pi_{2}\left(\pi_{1} z\right)\right)=0 \\
& \text { capacitor }:: N u m \rightarrow \text { SR }(\text { Pin } \times \text { Pin }) \\
& \text { capacitor }=\lambda c . \text { sigrel } z \text { where } \\
& \quad \text { twoPin } \diamond(z, u) \wedge \\
& c * \text { der } u=\pi_{2}\left(\pi_{1} z\right)
\end{aligned}
$$

Note that $c$ is a functional-level ( $\lambda$-bound) variable, while $u$ is a local signal variable.

A few differences may be noted compared to the earlier versions of these examples. The core syntax does not permit pattern matching; thus, projections are used to select from the signal interface variables. Additionally, compound equations are explicitly built up from atomic equations using $\wedge$ rather than appearing as a finite set, and connect-equations are assumed to have been desugared into atomic equations as explained in Sect. 2.2 as part of the translation into the core syntax.

\subsection{Equational Theory}

The equational theory ( $\beta \eta$-equality or conversion) for $\mu-\mathrm{H}$ is specified by an equivalence relation for each syntactic category: $\Gamma \vdash$ $t_{1} \simeq t_{2}: \tau$ for $\lambda$-terms, $\Gamma ; \Delta \vdash_{q} q_{1} \simeq q_{2}$ for equations, and $\Gamma ; \Delta \vdash_{s} s_{1} \simeq s_{2}: \sigma$ for signal expressions. Two terms that are equationally related are said to be convertible. The equational theory dictates the behaviour of our normalisation function; that is, a term should be convertible to its normal form, and convertible terms should have equal normal forms [10].
All three relations include the rules for reflexivity, symmetry, and transitivity to make them equivalence relations. The relation $\Gamma \vdash t_{1} \simeq t_{2}: \tau$ includes the the usual $\beta$ and $\eta$ rules of the $\lambda$ calculus and an additional $\eta$ rule for signal relations:

$$
\frac{\Gamma \vdash t: S R \sigma}{\Gamma \vdash t \simeq \operatorname{sigrel} z \text { where }(t \diamond z): S R \sigma} \quad(\eta \text {-SR })
$$

Note that $z$ is syntactically prohibited from occurring in $\Gamma$ or $t$.

The relation $\Gamma$; $\Delta \vdash_{q} q_{1} \simeq q_{2}$ for equations admits a $\beta$ rule for signal relations:

$$
\frac{\Gamma ; \epsilon, z: \sigma \vdash_{q} q \quad \Gamma ; \Delta \vdash_{s} s: \sigma}{\Gamma ; \Delta \vdash_{q}(\text { sigrel } z \text { where } q) \diamond s \simeq q[s / z]}
$$

Signal-level products were introduced to express $n$-ary signal relations. Hence, we need to reduce products, and the relation $\Gamma ; \Delta \vdash_{s}$ $s_{1} \simeq s_{2}$ is consequently the usual $\beta$ and $\eta$ rules for products. However, beyond this, no reductions are performed on signal expressions as the goal here is not to attempt to solve the equations.

Additionally, we make these equivalence relations into congruence relations by including the obvious congruence rules. For example, $\zeta$ for signal relations:

$$
\frac{\Gamma ; \Delta \vdash_{s} z_{1} \simeq z_{2}: \sigma \quad \Gamma ; \Delta \vdash_{q} q_{1} \simeq q_{2}}{\Gamma \vdash \text { sigrel } z_{1} \text { where } q_{1} \simeq \text { sigrel } z_{2} \text { where } q_{2}: S R \sigma}
$$

\section{Normalisation}

In this section, we give the semantics of $\mu-\mathrm{H}$ by describing how to reduce equational models to normal forms: essentially a flat set of equations along with information on how the system will evolve in response to any of the currently active switch conditions triggering (i.e., the signal in question crossing zero). We do so by appealing to Normalisation by Evaluation (NbE) [1].

There are two steps to using NbE. Firstly, terms are interpreted into a suitable mathematical model. We use the theorem prover Agda as our metalanguage, meaning we can encode our model directly as an Agda type. The second stage of $\mathrm{NbE}$ is then to take an object of the model and reify the object back into the normal form that it represents. Thus, the model can also be viewed as a set-theoretic denotation for our language. Moreover, by working in a constructive setting, the semantic objects in the model can be "evaluated", intimately relating the semantic specification and the implementation of the language.

A number of design decisions have been fixed by choosing $\mathrm{NbE}$. Our work could be viewed as a "deep embedding". One might then wonder why a simpler shallow embedding, one that directly exploits Agda's evaluation model, would not suffice (i.e., using higher-order abstract syntax). Ultimately, we do not want to rely on the unspecified semantics of a host language, particularly not a language as complex as Agda. Rather, we wish to define our semantics within the well-understood and thoroughly studied $\lambda$ calculus. Thus, a complementary, equally valid view is to consider our semantics as a translation of $\mu-\mathrm{H}$ into a suitable model.

Additional reasons for using $\mathrm{NbE}$ include the fact that $\mathrm{NbE}$ provides a reduction-free view of evaluation. This allows us to sidestep issues related to substitution. Avoiding a reduction-based approach also relieves us of the duty to prove reduction-based theorems, such as the first Church-Rosser theorem (confluence).

Our normalisation procedure is strong and produces canonical ( $\beta$-normal, $\eta$-long) normal forms. The normal forms contain no $\beta$ redexes, including those terms under binders, and are $\eta$-expanded as far as possible without introducing $\beta$-redexes. However, for a number of reasons that will be discussed in later sections, when branches are left unevaluated. As an example of canonical forms, consider normalising the identity function $\lambda f . f$ at the type $(\alpha \rightarrow$ $\beta) \rightarrow(\alpha \rightarrow \beta)$ to produce the canonical form $\lambda x . \lambda y . x y$. 


\subsection{Representation}

Using Agda as our metalanguage, we start by giving a representation for types, contexts, terms, and normal forms. Agda has a powerful termination checker, which is of use when formulating a complex system where termination and totality are important properties. Agda is also similar to other popular, contemporary functional languages, such as Haskell, thereby making the source code presented in this paper accessible to a wider audience.

Following Dybjer's notational conventions [12], we distinguish between object- and metalanguage concepts as follows:

- Alphabetic identifiers in the object-language are written in Sans Serif, whereas metalanguage constants are written in Roman.

- Object-language symbolic constants are denoted by using a dot above the corresponding metalanguage symbol. For example, the Agda function space is denoted $\rightarrow$, whereas the objectlanguage function space is denoted $\dot{\rightarrow}$. Similarly, we use a single dot for object-language application $(f \cdot x)$.

For the remainder of this section, the reader may find it useful to refer back to Sect. 3 to see the similarities between the syntax and typing relations, and the Agda definitions that represent them.

Types. Type represents functional level types $\tau$, while SType represents signal-level types $\sigma$ (see Fig. 4):

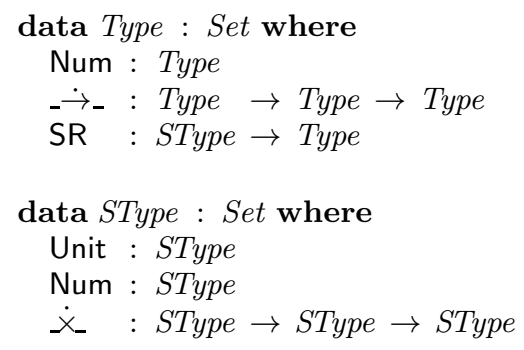

Contexts. We opt for a nameless approach to binding using de Bruijn indices [11] as this obviates the need for variable freshness conditions and makes $\alpha$-equivalent terms definitionally equivalent. Contexts are consequently implemented as parametric snoc lists and are used for both functional- and signal-level typing contexts:

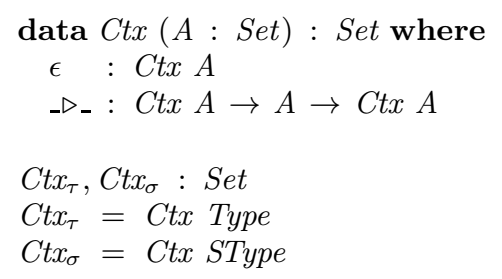

Contexts are not strictly required at the signal level as only one signal-level variable is ever in scope. Nevertheless, we opt for contexts at both levels for symmetry and to facilitate a refinement enforcing local signal variables to be well-scoped (see Sect. 7).

A variable is encoded as a de Bruijn index into a typing context. The constructor $z v$ points to the end of the context; i.e., the most recently bound variable. The constructor sv weakens a variable by extending the context into which it points. Intuitively, the type $\operatorname{VarIx} \Gamma \tau$ can be thought of as the set of indices pointing to variables of type $\tau$ in the context $\Gamma$ :

$$
\begin{aligned}
& \text { data VarIx : Ctx } A \rightarrow A \rightarrow \text { Set where } \\
& \text { zv : } \quad \operatorname{VarIx}(\Gamma \triangleright a) a \\
& \text { sv }: \operatorname{VarIx} \Gamma b \rightarrow \operatorname{VarIx}(\Gamma \triangleright a) b
\end{aligned}
$$

Terms. Term, STerm, and QTerm represent the functional-level terms ( $\lambda$-terms), signal-level terms, and equations, respectively.
They are indexed on contexts and type representations and correspond to the typing relations in Fig. 5 and Fig. 6: we are not just representing terms but typing derivations.

Interface variables (var) and local variables (Ivar) are distinguished in signal-level terms. As local variables are not subject to substitution or other computations during normalisation, a local variable in is simply represented by the anonymous constructor Ivar. In contrast, interface variables behave in very much the same manner as $\lambda$-bound variables. The fun constructor allows us to use functional level values of base type within signal expressions. For example, from Sect. 2, fun allows us to refer to the parameter $c$ in the equation $c * \operatorname{der} u=p . i$. For clarity, the parameters $\Gamma$ and $\Delta$ have been omitted from the constructors as they do not vary.

$$
\begin{aligned}
& \text { data } S T e r m\left(\Gamma: C t x_{\tau}\right)\left(\Delta: C t x_{\sigma}\right): \\
& (\sigma: \text { SType }) \rightarrow \text { Set where } \\
& \text { unit : STerm Unit } \\
& \text { Ivar : STerm Num } \\
& \text { var : VarIx } \Delta \sigma \rightarrow \text { STerm } \sigma \\
& \text { fun : Term Num } \rightarrow \text { STerm Num } \\
& \text { der : STerm Num } \rightarrow \text { STerm Num } \\
& \text { add : STerm Num } \rightarrow \text { STerm Num } \rightarrow \text { STerm Num } \\
& \text { sub : STerm Num } \rightarrow \text { STerm Num } \rightarrow \text { STerm Num } \\
& \text { mul : STerm Num } \rightarrow \text { STerm Num } \rightarrow \text { STerm Num } \\
& \text { pair : STerm } \sigma_{1} \rightarrow \operatorname{STerm} \sigma_{2} \rightarrow \operatorname{STerm}\left(\sigma_{1} \dot{\times} \sigma_{2}\right) \\
& \text { fst : STerm }\left(\begin{array}{lll}
\sigma_{1} & \dot{\times} & \sigma_{2}
\end{array}\right) \quad \rightarrow \text { STerm } \sigma_{1} \\
& \text { snd : } \operatorname{STerm}\left(\begin{array}{lll}
\sigma_{1} & \dot{\times} & \sigma_{2}
\end{array}\right) \quad \rightarrow \text { STerm } \sigma_{2}
\end{aligned}
$$

Equations are represented in a straight-forward manner following Sect. 3; parameters are again omitted where they are obvious:

$$
\begin{aligned}
& \text { data } Q \operatorname{Term}\left(\Gamma: C t x_{\tau}\right)\left(\Delta: C t x_{\sigma}\right): \text { Set where } \\
& \text { empty : QTerm } \\
& \wedge_{-}: \text {QTerm } \rightarrow \text { QTerm } \rightarrow \text { QTerm } \\
& \diamond_{-} \quad: \operatorname{Term}(S R \sigma) \rightarrow \text { STerm } \sigma \rightarrow \text { QTerm } \\
& { }_{-}{ }_{-} \quad: S T e r m \sigma \rightarrow S T e r m \sigma \rightarrow \text { QTerm } \\
& \text { switch : Switch } \rightarrow \text { QTerm } \\
& \text { data Switch }\left(\Gamma: C t x_{\tau}\right)\left(\Delta: C t x_{\sigma}\right): \text { Set where } \\
& \text { initially : QTerm } \rightarrow \text { Switch } \\
& \text { _when_ } \Rightarrow \text { _: Switch } \rightarrow \text { STerm Num } \\
& \rightarrow \text { QTerm } \rightarrow \text { Switch }
\end{aligned}
$$

Finally, the $\lambda$-terms can be defined. For numeric literals (lit), a type of real numbers is postulated and embedded in the representation:

$$
\begin{aligned}
& \text { data } \operatorname{Term}\left(\Gamma: C t x_{\tau}\right):(\tau: \text { Type }) \rightarrow \text { Set where } \\
& \text { unit : Term } \Gamma \text { Unit } \\
& \text { lit : } \mathbb{R} \rightarrow \text { Term } \Gamma \text { Num } \\
& \text { var : VarIx } \Gamma \tau \quad \rightarrow \text { Term } \Gamma \tau \\
& \text {-- } \quad: \operatorname{Term} \Gamma\left(\tau_{1} \dot{\rightarrow} \tau_{2}\right) \rightarrow \operatorname{Term} \Gamma \tau_{1} \\
& \rightarrow \text { Term } \Gamma \tau_{2} \\
& \text { lam : } \operatorname{Term}\left(\Gamma \triangleright \tau_{1}\right) \tau_{2} \rightarrow \operatorname{Term} \Gamma\left(\tau_{1} \rightarrow \tau_{2}\right) \\
& \text { sigrel : QTerm } \Gamma(\epsilon \triangleright \sigma) \rightarrow \operatorname{Term} \Gamma(S R \sigma)
\end{aligned}
$$

Hydra re-revisited. Once again, it is useful to revisit the examples presented earlier in the paper:

$$
\begin{aligned}
& \text { Pin : SType } \\
& \text { Pin }=\text { Num } \dot{\times} \text { Num } \\
& \text { twoPin : Term } \Gamma(\text { SR }((\text { Pin } \dot{\times} \text { Pin }) \dot{\times} \text { Num })) \\
& \text { twoPin }=\text { sigrel }( \\
& \quad \text { fst }(\text { fst }(\text { fst } z))-\text { fst }(\text { snd }(\text { fst } z)) \doteq \text { snd } z \wedge \\
& \text { snd }(\text { fst }(\text { fst } z))+\text { snd }(\text { snd }(\text { fst } z)) \doteq \text { fun }(\text { lit } 0)) \\
& \quad \text { where } z=\operatorname{var} \text { zv }
\end{aligned}
$$




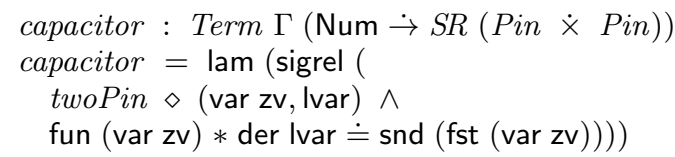

The main difference in the above representation compared with the abstract core syntax is the use of well-scoped, nameless variables, both at the functional level and signal level. In contrast, the positions of local variables in signal terms are now only tagged with the anonymous constructor Ivar rather than being identified.

Normal and Neutral Terms. One could reuse the Term representation for normal forms: after all, the normal forms are a subset of the terms. However, we choose to use a datatype of canonical terms that is guaranteed by construction to only permit $\beta$-normal $\eta$-long terms, paired with an embedding back into terms.

The approach is to stratify the canonical term representation such that only terms that are free of $\beta$-redexes can be constructed. Furthermore, by only permitting variables to occur at base types in normal forms, the representation guarantees that canonical terms are fully $\eta$-expanded. There are two kinds of strata (layers): Normal and Neutral. Normal canonical terms are those that have been computed as far as possible constructor. Neutral canonical terms those that are stuck by a variable in a key position.

$\mathrm{SNeu}$ and $\mathrm{SNrm}$ represent the neutral and normal strata of signal-level terms. A number of these terms, such as der and add, could have been considered normal instead of neutral (as they are uninterpreted). However, as normal terms by convention are taken to be only terms in constructor form, we opted to include said terms in $S N e u$ for consistency. The type Base is a predicate on (signal) Types that is inhabited only when applied to base types (i.e: Unit and Num). As before, constant parameters are omitted from constructors:

$$
\begin{aligned}
& \text { data } \operatorname{SNeu}\left(\Gamma: C t x_{\tau}\right)\left(\Delta: C t x_{\sigma}\right): \\
& (\sigma: \text { SType }) \rightarrow \text { Set where } \\
& \text { Ivar : SNeu Num } \\
& \text { var : } \operatorname{VarIx} \Delta \sigma \rightarrow \text { SNeu } \sigma \\
& \text { der : SNrm Num } \rightarrow \text { SNeu Num } \\
& \text { add }: S N r m \text { Num } \rightarrow \text { SNrm Num } \rightarrow \text { SNeu Num } \\
& \text { sub : SNrm Num } \rightarrow \text { SNrm Num } \rightarrow \text { SNeu Num } \\
& \text { mul }: S N r m \text { Num } \rightarrow \text { SNrm Num } \rightarrow \text { SNeu Num } \\
& \text { fst }: S N e u\left(\sigma_{1} \dot{x} \sigma_{2}\right) \rightarrow \text { SNeu } \sigma_{1} \\
& \text { snd : } \operatorname{SNeu}\left(\begin{array}{lll}
\sigma_{1} & \dot{\times} & \sigma_{2}
\end{array}\right) \rightarrow \text { SNeu } \sigma_{2} \\
& \text { data } \operatorname{SNrm}\left(\Gamma: C t x_{\tau}\right)\left(\Delta: C t x_{\sigma}\right): \\
& (\sigma: \text { SType }) \rightarrow \text { Set where } \\
& \text { unit : } S N r m \text { Unit } \\
& \text { fun : Nrm Num } \rightarrow \text { SNrm Num } \\
& \text { pair : } S N r m \sigma_{1} \rightarrow \operatorname{SNrm} \sigma_{2} \rightarrow \operatorname{SNrm}\left(\sigma_{1} \dot{\times} \sigma_{2}\right) \\
& \text { neu : Base } \sigma \rightarrow \text { SNeu } \sigma \rightarrow \text { SNrm } \sigma
\end{aligned}
$$

Equations do not require stratification as there are no directly inductive cases that can be reduced during normalisation. QNeu and SwitchNeu both represent normalised equations. We omit the definition of $Q N e u$ as it is structurally the same as QTerm, with Term replaced by $N e u, S T e r m$ replaced by $S N r m$, and Switch replaced by SwitchNeu. SwitchNeu is given below:

$$
\begin{aligned}
& \text { data SwitchNeu }\left(\Gamma: C t x_{\tau}\right)\left(\Delta: C t x_{\sigma}\right) \text { : Set where } \\
& \text { initially : QNeu } \rightarrow \text { SwitchNeu } \\
& \text { _when_ } \Rightarrow_{-}: \text {SwitchNeu } \rightarrow \text { SNrm Num } \\
& \rightarrow \text { QTerm } \rightarrow \text { SwitchNeu }
\end{aligned}
$$

The initially fragment will become active immediately (i.e., as part of the current generation of equations to be simulated) and thus needs to contain a flat set of equations; hence the use of $Q N e u$. By contrast, for reasons that will be discussed later, the equations of when-branches should not be normalised prematurely; hence QTerm. The conditions of the branches will, however, become active and thus need to be normalised; hence $S N r m$ Num.

Finally, the stratifictaion of functional-level terms is as follows:

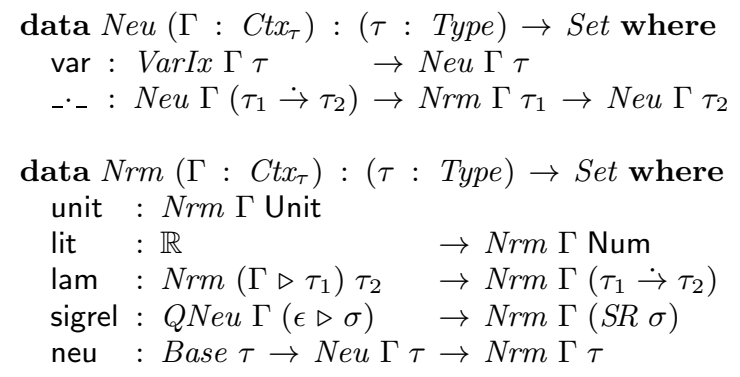

\subsection{The Model}

An important aspect of $\mathrm{NbE}$ is the reification of objects in the model to normal forms. The normal forms must be $\eta$-long. Thus, the process of reification may build a sub-term in a context extended by new variables. For example, the term $\lambda f . f$ of type $\left(\tau_{1} \rightarrow \tau_{2}\right) \rightarrow$ $\left(\tau_{1} \rightarrow \tau_{2}\right)$ will be expanded to $\lambda f . \lambda x . f x$, which constructs the sub-term $f x$ in a context extended by the new variable $x$. This behaviour is accounted for in the model using context morphisms.

A context morphism is a preorder (a reflexive and transitive binary relation) specified by the type $\Gamma_{2} \sqsupseteq \Gamma_{1}$ to denote a morphism from the context $\Gamma_{1}$ to $\Gamma_{2}$. One can view morphisms as a general mechanism for weakening contexts. The symbol $\sqsupseteq$ is chosen to emphasise that the context on the left is in some sense weaker than the context appearing on the right, i.e., any term that is well-scoped in $\Gamma_{1}$ is also well-scoped in $\Gamma_{2}$ by weakening.

$$
\begin{aligned}
& \text { data }{ }_{-}: \text {Ctx } A \rightarrow C t x A \rightarrow \text { Set where } \\
& \epsilon \quad: \Gamma_{\epsilon} \sqsupseteq \epsilon \\
& \triangleright_{-}: \Gamma_{2} \sqsupseteq \Gamma_{1} \rightarrow \operatorname{VarIx} \Gamma_{2} a \rightarrow \Gamma_{2} \sqsupseteq\left(\Gamma_{1} \triangleright a\right)
\end{aligned}
$$

There is a morphism from the empty context $(\epsilon)$ to every other context. Additionally, any morphism between contexts can be extended $\left(\_\triangleright_{-}\right)$on the right provided there is a corresponding variable in the target context.

The reader may have noticed that context morphisms correspond exactly to the notion of Kripke relations [22] from model theory. In this sense, we are building a Kripke-style model for our language. This should not come as a surprise given the connection between $\lambda$-calculi and intuitionistic logic.

Morphisms are isomorphic to functions on indices (proof omitted, see [5]); one may convert between the two using the maps given below. However, representing morphisms as data has a few advantages, namely, one may pattern match on morphisms in Agda. Below, curly braces $(\{\})$ denote implicit arguments - arguments that need not be supplied when it is possible to infer them from context:

$$
\begin{aligned}
& \text { toMorph: }\left(\operatorname{VarIx} \Gamma_{1} \tau \rightarrow \operatorname{VarIx} \Gamma_{2} \tau\right) \rightarrow \Gamma_{2} \sqsupseteq \Gamma_{1} \\
& \text { toMorph }\left\{\Gamma_{1}=\epsilon\right\} \quad f=\epsilon \\
& \text { toMorph }\left\{\Gamma_{1}=\Gamma \triangleright \tau\right\} f=\text { toMorph }(f \circ \mathrm{sv}) \triangleright f \mathrm{zv} \\
& \text { fromMorph }: \Gamma_{2} \sqsupseteq \Gamma_{1} \rightarrow\left(\operatorname{VarIx} \Gamma_{1} \tau \rightarrow \operatorname{VarIx} \Gamma_{2} \tau\right) \\
& \text { fromMorph } \epsilon \quad() \\
& \text { fromMorph }(\Gamma \triangleright x) \mathrm{zv}=x \\
& \text { fromMorph }(\Gamma \triangleright x)(\mathrm{sv} i)=\text { fromMorph } \Gamma i
\end{aligned}
$$

By using a context morphism to relate variable indices one is able to weaken terms, normal forms, and environments. A context morphism is necessarily reflexive and transitive, and hence a preorder: 


$$
\begin{aligned}
& \text { reft }: \Gamma \sqsupseteq \Gamma \\
& \text { reft }=\text { toMorph id } \\
& \text { trans }: \Gamma_{3} \sqsupseteq \Gamma_{2} \rightarrow \Gamma_{2} \sqsupseteq \Gamma_{1} \rightarrow \Gamma_{3} \sqsupseteq \Gamma_{1} \\
& \text { trans } \phi \psi=\text { toMorph }(\text { fromMorph } \phi \circ \text { fromMorph } \psi \text { ) }
\end{aligned}
$$

Furthermore, it is easy to define weakening under binders or variable exchange by providing the appropriate morphism:

$$
\begin{aligned}
& \text { step }:(\Gamma \triangleright a) \sqsupseteq \Gamma \\
& \text { step }=\text { toMorph sv } \\
& \text { swap : }\left(\Gamma \triangleright \tau_{1} \triangleright \tau_{2}\right) \sqsupseteq\left(\Gamma \triangleright \tau_{2} \triangleright \tau_{1}\right) \\
& \text { swap }=\text { toMorph }(\mathrm{sv} \circ \mathrm{sv}) \triangleright \mathrm{zv} \triangleright \mathrm{sv} \mathrm{zv}
\end{aligned}
$$

The model itself is specified by a mapping from our representation types into Agda types. $S \mathrm{Val}$ defines the type of signal-level values in the model:

$$
\begin{aligned}
& \text { SVal : Ctx } x_{\tau} \rightarrow C t x_{\sigma} \rightarrow \text { SType } \rightarrow \text { Set } \\
& \text { SVal } \Gamma \Delta \text { Unit } \quad=S N r m \Gamma \Delta \text { Unit } \\
& S \text { Val } \Gamma \Delta \text { Num } \quad=S N r m \Gamma \Delta \text { Num } \\
& S \operatorname{Val} \Gamma \Delta\left(\begin{array}{lll}
\sigma_{1} \dot{\times} & \sigma_{2}
\end{array}\right)=S \operatorname{Val} \Gamma \Delta \sigma_{1} \times S \operatorname{Val} \Gamma \Delta \sigma_{2}
\end{aligned}
$$

Values of type Unit and Num are represented by their normal forms. Signal-level products are represented by Agda products of signal values.

At the functional level, function types are recognised as Agda function spaces between values with the aforementioned context morphism. This morphism will make the job of interpretation simple when moving under binders. Signal relations are also transformed into metalevel functions from signal values into sets of equations in a normal form. Signal relation values also require a morphism as functional-level terms may appear in the body of a signal relation using the fun constructor.

$$
\begin{aligned}
& \text { QVal : Ctx } x_{\tau} \rightarrow C t x_{\sigma} \rightarrow \text { Set } \\
& Q \mathrm{Val}=\mathrm{QNeu} \\
& \text { Val : Ctx } \rightarrow \text { Type } \rightarrow \text { Set } \\
& \text { Val } \Gamma_{1} \text { Unit } \quad=N r m \Gamma_{1} \text { Unit } \\
& \text { Val } \Gamma_{1} \text { Num } \quad=N r m \Gamma_{1} \text { Num } \\
& \text { Val } \Gamma_{1}\left(\tau_{1} \dot{\rightarrow} \tau_{2}\right)=\forall\left\{\Gamma_{2}\right\} \quad \rightarrow \Gamma_{2} \sqsupseteq \Gamma_{1} \\
& \rightarrow \operatorname{Val} \Gamma_{2} \tau_{1} \rightarrow \operatorname{Val} \Gamma_{2} \tau_{2} \\
& \text { Val } \Gamma_{1}(\mathrm{SR} \sigma) \quad=\forall\left\{\Gamma_{2} \Delta\right\} \quad \rightarrow \Gamma_{2} \sqsupseteq \Gamma_{1} \\
& \rightarrow \operatorname{SVal} \Gamma_{2} \Delta \sigma \rightarrow Q \operatorname{Val} \Gamma_{2} \Delta
\end{aligned}
$$

\subsection{Interpretation}

We are now almost ready to give an interpreter to map terms into semantic objects. However, first we must introduce a notion of environments. An environment stores the values of variables required for substitution. Environments are implemented as snoc lists and are indexed by two contexts. The first context is the context of values occuring in the environment. The second indexing context fixes the length of the environment by requiring a value for each of the variables it contains. Using two contexts rather than a single context for both tasks makes the definition more general, allowing values in the environment to be weakened without changing the length of the environment.

$$
\begin{aligned}
& \text { data } E n v: C t x_{\tau} \rightarrow C t x_{\tau} \rightarrow \text { Set where } \\
& \quad \epsilon \quad: \text { Env } \Gamma \epsilon \\
& \quad{ }_{-}{ }_{-}: \text {Env } \Gamma_{1} \Gamma_{2} \rightarrow \operatorname{Val} \Gamma_{1} \tau \rightarrow \operatorname{Env} \Gamma_{1}\left(\Gamma_{2} \triangleright \tau\right)
\end{aligned}
$$

Environments are either empty $(\epsilon)$, or may be extended by a new value (_ $\left.\triangleright_{-}\right)$. Values may then be retrieved from the environment using lookup:

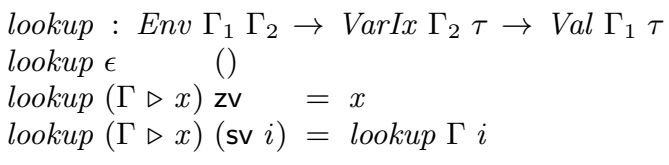

Signal-level environments (SEnv) are defined in much the same way with an additional functional-level context parameter, needed for the occurrence of $S \mathrm{Val}$, which replaces $\mathrm{Val}$ in the above definition of environments.

Environments and values suffice to define our model:

$$
\begin{aligned}
& \text { Model : Ctx } \rightarrow \text { Type } \rightarrow \text { Set } \\
& \text { Model } \Gamma_{1} \tau=\forall\left\{\Gamma_{2}\right\} \\
& \rightarrow \quad \operatorname{Env} \Gamma_{2} \Gamma_{1} \\
& \rightarrow \quad \operatorname{Val} \Gamma_{2} \tau \\
& \text { QModel : Ctx } x_{\tau} \rightarrow C t x_{\sigma} \rightarrow \text { Set } \\
& \text { QModel } \Gamma_{1} \Delta_{1}=\forall\left\{\Gamma_{2} \Delta_{2}\right\} \\
& \rightarrow \text { Env } \quad \Gamma_{2} \Gamma_{1} \\
& \rightarrow \text { SEnv } \Gamma_{2} \Delta_{2} \Delta_{1} \\
& \rightarrow Q \text { Val } \Gamma_{2} \Delta_{2} \\
& \text { SModel : Ctx } x_{\tau} \rightarrow \text { Ctx } x_{\sigma} \rightarrow \text { SType } \rightarrow \text { Set } \\
& \text { SModel } \Gamma_{1} \Delta_{1} \sigma=\forall\left\{\Gamma_{2} \Delta_{2}\right\} \\
& \rightarrow \operatorname{Env} \quad \Gamma_{2} \Gamma_{1} \\
& \rightarrow \text { SEnv } \Gamma_{2} \Delta_{2} \Delta_{1} \\
& \rightarrow \mathrm{SVal} \quad \Gamma_{2} \Delta_{2} \sigma \\
& \text { SwitchModel : Ctx } x_{\tau} \rightarrow C t x_{\sigma} \rightarrow \text { Set } \\
& \text { SwitchModel } \Gamma_{1} \Delta_{1}=\forall\left\{\Gamma_{2} \Delta_{2}\right\} \\
& \rightarrow \operatorname{Env} \Gamma_{2} \Gamma_{1} \\
& \rightarrow \operatorname{SEnv} \Gamma_{2} \Delta_{2} \Delta_{1} \\
& \rightarrow \text { SwitchNeu } \Gamma_{2} \Delta_{2}
\end{aligned}
$$

We can now define an interpreter from terms into our model at both levels of the language. We begin with the interpretation of signal-level terms:

$$
\begin{aligned}
& \llbracket \cdot \rrbracket_{s}: \text { STerm } \Gamma \Delta \sigma \rightarrow \text { SModel } \Gamma \Delta \sigma \\
& \text { 【lvar } \quad \rrbracket_{s} \rho e=\text { neu Ivar } \\
& \llbracket \operatorname{var} z \quad \rrbracket_{s} \rho e=\text { lookup ez } \\
& \text { 【fun } t \quad \prod_{s} \rho e=\text { fun }(\llbracket t \rrbracket \rho) \\
& \text { unit } \rrbracket_{s} \rho e=\text { unit } \\
& \llbracket \operatorname{der} s \quad \rrbracket_{s} \rho e=\operatorname{neu}\left(\operatorname{der}\left(\llbracket s \rrbracket_{s} \rho e\right)\right) \\
& \llbracket \operatorname{add} s_{1} s_{2} \rrbracket_{s} \rho e=\text { neu }\left(\text { add }\left(\llbracket s_{1} \rrbracket_{s} \rho e\right)\left(\llbracket s_{2} \rrbracket_{s} \rho e\right)\right) \\
& \llbracket \operatorname{sub} s_{1} s_{2} \rrbracket_{s} \rho e=\operatorname{neu}\left(\operatorname{sub}\left(\llbracket s_{1} \rrbracket_{s} \rho e\right)\left(\llbracket s_{2} \rrbracket_{s} \rho e\right)\right) \\
& \llbracket \text { mul } s_{1} s_{2} \rrbracket_{s} \rho e=\operatorname{neu}\left(\operatorname{mul}\left(\llbracket s_{1} \rrbracket_{s} \rho e\right)\left(\llbracket s_{2} \rrbracket_{s} \rho e\right)\right) \\
& \llbracket \text { pair } s_{1} s_{2} \rrbracket_{s} \rho e=\left(\llbracket s_{1} \rrbracket_{s} \rho e\right),\left(\llbracket s_{2} \rrbracket_{s} \rho e\right) \\
& \text { 『fst } s \quad \prod_{s} \rho e=\pi_{1}\left(\llbracket s \rrbracket_{s} \rho e\right) \\
& \text { 【snd } s \quad \prod_{s} \rho e=\pi_{2}\left(\llbracket s \rrbracket_{s} \rho e\right)
\end{aligned}
$$

The interpretation function takes three arguments: a signal-level term of type $\sigma$ in a context $\Delta$; a functional-level environment (to be passed on when we encounter the fun constructor); and an environment that provides a value (in some possibly extended context), for each variable in $\Delta$. The function then returns a value in our model of the appropriate type in the extended context.

To interpret signal-level variables, we simply find their value in the environment. Of course, a term may be open in that it may contain a well-scoped variable that is not $\lambda$-bound. For example, in a context $\left(\epsilon \triangleright \tau_{1}\right)$, the $\lambda$-term $(\lambda x . y)$ would be represented lam (var (sv zv)) (as $y$ must refer to the only non- $\lambda$-bound variable in scope) with type Term $\left(\epsilon \triangleright \tau_{1}\right)\left(\tau_{2} \dot{\rightarrow} \tau_{1}\right)$. To account for unbound variables of this nature, a variable that is unbound but well-scoped with respect to an environment is considered a 
value under that environment. This ensures totality of lookup and guarantees that open variables are put into an $\eta$-long form.

For signal-level products, we interpret them into Agda products, where,$_{-}$constructs a product in our model, and $\pi_{1}$ and $\pi_{2}$ are the eliminators.

$$
\begin{aligned}
& \llbracket \cdot \rrbracket: \text { Term } \Gamma \tau \rightarrow \text { Model } \Gamma \tau \\
& \llbracket \text { unit } \rrbracket \rho=\text { unit } \\
& \llbracket \text { lit } n \quad \prod \rho=\text { lit } n \\
& \llbracket \operatorname{var} x \quad \rrbracket \rho=\text { lookup } \rho x \\
& \llbracket f \cdot x \rrbracket \rho=(\llbracket f \rrbracket \rho) \text { refl }(\llbracket x \rrbracket \rho) \\
& \llbracket \text { lam } t \rrbracket \rho=\lambda \phi x \rightarrow \llbracket t \rrbracket(\text { wknEnv } \phi \rho \triangleright x) \\
& \llbracket \text { sigrel } q \rrbracket \rho=\lambda \phi x \rightarrow \llbracket q \rrbracket_{q}(\text { wknEnv } \phi \rho)(\epsilon \triangleright x)
\end{aligned}
$$

Function application proceeds by interpreting the function $f$ into an Agda function, which can then be applied to the interpretation of the argument $x$. For function types we are also required to provide a context morphism argument, which in this instance, is the identity morphism refl. One might wonder when context morphisms are actually required, given the above usage of refl. However, as we shall see with abstraction, they are put to work in weakening the environment $\rho$ using $w k n E n v$ :

$$
\text { wknEnv : } \Gamma_{2} \sqsupseteq \Gamma_{1} \rightarrow E n v \Gamma_{1} \Gamma \rightarrow E n v \Gamma_{2} \Gamma
$$

Furthermore, we will see examples when this morphism is required to be non-trivial, for examples, during reification.

Abstraction requires us to produce a function in our model. This is achieved by introducing the two arguments; a morphism and the value over which we are abstracting. The return value is then just the interpretation of the body of the abstraction, with the environment extended by the bound variable. Note the requirement to weaken the environment $\rho$, as we are moving under a binder.

Signal relations are treated in a similar way as $\lambda$-abstractions. A singleton environment of the bound signal variables is passed to the equation interpretation.

$$
\begin{aligned}
& \llbracket \cdot \rrbracket_{q}: \text { QTerm } \Gamma \Delta \rightarrow \text { QModel } \Gamma \Delta \\
& \text { 【empty } \rrbracket_{q} \rho e=\text { empty } \\
& \llbracket q_{1} \wedge q_{2} \rrbracket_{q} \rho e=\llbracket q_{1} \rrbracket_{q} \rho e \wedge \llbracket q_{2} \rrbracket_{q} \rho e \\
& \llbracket s r \diamond s \rrbracket_{q} \rho e=\llbracket s r \rrbracket \rho \text { refl }\left(\llbracket s \rrbracket_{s} \rho e\right) \\
& \llbracket s_{1} \doteq s_{2} \rrbracket_{q} \rho e=\text { reify }_{s}\left(\llbracket s_{1} \rrbracket_{s} \rho e\right) \doteq \text { reify }_{s}\left(\llbracket s_{2} \rrbracket_{s} \rho e\right) \\
& \llbracket \text { switch } w \rrbracket_{q} \rho e=\operatorname{switch}\left(\llbracket w \rrbracket_{s w} \rho e\right)
\end{aligned}
$$

For equations, interpretation of empty equations and pairing is done in the obvious structural way. Interpretation of signal relation application follows the same principles as standard function application. For the interpretation of atomic equations, recall that $\mathrm{NbE}$ proceeds in two steps: terms are interpreted into the model and then reified back into normal forms. Here, signal values have to be coerced back into normal forms using reifys because of the two-level nature of the core language. Details on reification will be deferred until the next section.

$$
\begin{aligned}
& \llbracket \cdot \rrbracket_{s w}: \text { Switch } \Gamma \Delta \rightarrow \text { SwitchModel } \Gamma \Delta \\
& \left.\llbracket \text { initially } \quad q \rrbracket_{s w} \rho e=\text { initially } \mathbb{\llbracket} q \rrbracket_{q} \rho e\right) \\
& \llbracket w \text { when } s \Rightarrow q \rrbracket_{s w} \rho e=\left(\llbracket w \rrbracket_{s w} \rho e\right) \text { when } \\
& \qquad \begin{array}{l}
\left.\llbracket s \rrbracket_{s} \rho e\right) \Rightarrow\left(\text { subst }_{q} q \rho e\right)
\end{array}
\end{aligned}
$$

The final component of interpretation is to handle switches. A family of substitution functions (subst) performs substitution of variables bound outside when-branches, leaving the body of said branches otherwise unnormalised. Substitution is required as the body of a when-branch may mention variables that are bound and applied in the enclosing context. It is important to leave whenbranches unnormalised for three reasons:

1. Operationally, premature normalisation of a branch is in general wasteful as a branch may never be activated. Leaving when- branches unnormalised thus reflects what an implementation should do.

2. To address the issue of transfer of simulation state across switches (see Sect. 7), it is necessary to allow the normalisation to depend on state information at the time of a switch, by allowing references to signal values just prior to a switch event, or by events carrying a payload. Thus, once such functionality is added to the language, it will not in general be possible to normalise a branch before it is switched in.

3. For many real-world applications, it is also desirable, or even essential, to allow equations to be computed by tail-calling a signal relation, guarding the recursive calls by a when-branch (for example, consider modelling a state machine). Postponing normalisation of when-branches permits a form of guarded corecursion, allowing such calls to be productive.

(The switch semantics already allows for switching back and forth between operational modes. However, recursive signal relations would add considerable convenience and flexibility.)

\subsection{Reification}

With interpretation defined, terms can be mapped to appropriate objects in the model. All that remains is to translate these objects back into the normal forms that represent them. The translation of values back into normal forms is often called reification. Reification is important for producing $\eta$-long normal forms, as can be seen in the definition for signal values:

$$
\begin{aligned}
& \text { reify }_{s}:(\sigma: \text { SType }) \rightarrow \text { SVal } \Gamma \Delta \sigma \rightarrow \text { SNrm } \Gamma \Delta \sigma \\
& \text { reify Unit } v=v \\
& \text { reify } \text { Num } v=v \\
& \text { reify }_{s}\left(\sigma_{1} \dot{\times} \sigma_{2}\right)(v, w)=\operatorname{pair}\left(\text { reify }_{s} \sigma_{1} v\right)\left(\text { reify }_{s} \sigma_{2} w\right)
\end{aligned}
$$

Reification is type directed. At base types signal values are already normal forms; conversely, product types must be built using the pair constructor. Similarly, at the functional level, signal relations must be constructed from the sigrel constructor, which guarantees that values of type SR Unit with no free variables must be a flat set of equations.

$$
\begin{aligned}
& \text { reify : }(\tau: \text { Type }) \rightarrow \text { Val } \Gamma \tau \rightarrow \text { Nrm } \Gamma \tau \\
& \text { reify Unit } \quad v=v \\
& \text { reify Num } \quad v=v \\
& \text { reify }(\mathrm{SR} \sigma) \quad f=\text { sigrel }\left(f \text { refl }\left(\text { reflect }_{s} \sigma(\text { var zv })\right)\right) \\
& \text { reify }\left(\tau_{1} \rightarrow \tau_{2}\right) f=\text { lam }\left(\text { reify } \tau_{2}(f \text { step }\right.
\end{aligned}
$$$$
\left.\left.\left(\text { reflect } \tau_{1}(\operatorname{var} z \mathbf{v})\right)\right)\right)
$$

For function spaces and signal relations we construct the $\eta$ expanded term by applying the Agda function that models the value to the variable just bound by the enclosing outer constructor (i.e., the term $f$ is mapped to $\lambda x$. $(f x)$ ). However, $f$ is a map on values, but variables are neutral terms. Thus, an inverse of reification, often called reflection, is required to take neutral terms to values.

$$
\begin{aligned}
& \text { reflect }:(\tau: \text { Type }) \rightarrow \text { Neu } \Gamma \tau \rightarrow \text { Val } \Gamma \tau \\
& \text { reflect } \operatorname{Num} \quad n=\text { neu Num } n \\
& \text { reflect }(\operatorname{SR} \sigma) \quad f=\lambda \phi v \rightarrow \text { wknNeu } \phi f \diamond \text { reify } \sigma v \\
& \text { reflect }\left(\tau_{1} \rightarrow \tau_{2}\right) f=\lambda \phi v \rightarrow \\
& \quad \text { reflect } \tau_{2}\left(\text { wknNeu } \phi f \cdot \text { reify } \tau_{1} v\right)
\end{aligned}
$$

We are finally ready to define our normalisation procedure. The function embed is taken to be the embedding of normal forms back into terms, and $i d E n v$ is the environment containing a value representation for each variable in the context:

norm : Term $\Gamma \tau \rightarrow$ Term $\Gamma \tau$

norm $t=$ embed $($ reify $\tau(\llbracket t \rrbracket i d E n v))$ 


\subsection{Summary and Properties}

In this section, a normalisation procedure has been developed for one iteration of the discrete aspect of the core language. The procedure has a number of desirable properties:

- Strong: the procedure produces canonical normal forms.

- Termination and totality: combined with the previous remark, termination gives us a guarantee that all well-typed programs of type SR Unit result in a flat set of equations. To the authors' knowledge, this is a new result for structurally dynamic modular systems of equations.

- Type preserving: the procedure preserves the types of expression during normalisation.

- Efficiency: compared to a more direct implementation (e.g., via a shallow embedding), the procedure is relatively efficient. Furthermore, through standard optimisation techniques, $\mathrm{NbE}$ can be made to rival the efficiency of the underlying metalanguage [2]. While the metalanguage currently used is not particularly efficient, one could extract/transcribe the procedure into a more efficient functional language (e.g. Haskell).

- Independence: normalisation has been specified in a way that is independent of the continuous semantics, allowing the continuous aspects to be specified in a way that is appropriate for a particular domain.

\section{Structural Dynamism}

Section 2 introduced the concept of a structurally dynamic system. We now make a first attempt at formally describing the semantics of such systems. When a signal relation without any free variables is normalised, the result is essentially a flat system of equations: all signal relation applications have been eliminated. Some of these equations are the initially active ones. Others may become active later, should the when-condition guarding them become true, causing previously active equations from the same switch to be deactivated. As we wish to keep the semantics of the continuous aspects separate, we appeal to an external simulation oracle for the meaning of the active equations and when-conditions.

The interaction with the oracle naturally separates into two parts: extraction of initially active equations and conditions to be given to the oracle, and processing of events received from the oracle, determined by the semantics of the active equations and conditions and resulting in an updated system configuration.

The extraction function has the following type:

$$
\begin{aligned}
\text { extract }: & \text { Nrm } \epsilon(\text { SR Unit }) \\
\rightarrow & (\text { FlatEqns } \times \text { FlatEqns } \times \text { Conds })
\end{aligned}
$$

Given the normal form of a nullary signal relation, extract returns the active equations as a set of atomic equations, a set of corresponding atomic initialisation equations, and a set of active whenconditions. Each condition is labelled by a distinct natural number to relate it to its originating when-branch. The fact that extract is defined on $N r m \in$ (SR Unit) ensures that extraction is total: we know statically that extract will never encounter a signal relation application, even though a cursory inspection of the abstract syntax might suggest otherwise.

The event processing function has the following type:

$$
\begin{aligned}
\text { processEvents }: & \text { Nrm } \epsilon(\text { SR Unit }) \rightarrow \text { List } \mathbb{N} \\
& \rightarrow \text { Term } \epsilon \text { (SR Unit) }
\end{aligned}
$$

Given the current system configuration and a list of events that have occurred at a specific point in time, each identified by the label of the corresponding when-condition and thus linked to a specific when-branch, a new system configuration is computed from which in turn new sets of equations and conditions can be extracted for continued simulation.

To illustrate the idea, consider the simple Hydra program given below which has only two structural configurations. Two equations have been tagged as initialisation (init) and re-initialisation (reinit) equations, and the pre function is used to allow state transfer between configurations (i.e., pre $x$ refers to the instantaneous value of $x$ prior to the structural change):

$$
\begin{aligned}
& \text { model }: \text { SR Num } \\
& \text { model }=\text { sigrel } x \text { where } \\
& \text { init } x=0 \\
& \text { reinit } x=\text { pre } x \\
& y=x * x \\
& \text { initially } \Rightarrow \\
& \text { der } x=1 \\
& \text { when }(\text { time }-10) \Rightarrow \\
& \text { der } x=-0.5
\end{aligned}
$$

When simulation begins, extract returns a triple of the atomic equations (left), the intialisation equations (centre), and the switch conditions (right):

$$
\begin{array}{ll}
y & =x * x \\
\operatorname{der} x & =1
\end{array} \quad x=0 \quad \text { time }-10
$$

At time 10 , when the switch condition time -10 crosses 0 , processEvents is invoked. It determines which switch branches should be active and creates a term by selecting equations from the active branches and mapping reinit equations to init equations. This term is then normalised. Thus, at the start of the second structural configuration, extract would produce the following sets of atomic equations, initialisation equations, and switch conditions. Note that the switch condition, while still active, will never fire as it will not cross 0 again:

$$
\begin{array}{ll}
y & =x * x \\
\operatorname{der} x & =-0.5
\end{array} \quad x=\text { pre } x \quad \text { time }-10
$$

The normalisation and processing of events can be iterated, possibly indefinitely, in response to the triggering of events during simulation run-time. For example, consider modelling elastic collisions of bodies in a closed system, using event switches at the time of a collision to compute new trajectories for the bodies. To handle a potentially infinite number of equation systems we represent the procedure coinductively. A CoList of sets of equations is generated by normalising an input term, extracting the initial equations, and then corecursively computing the following systems of equations using the functions defined above and the simulation oracle. This is achieved using Agda's native support for coinduction [24].

Our current formalisation does not take explicit account of the way state is transferred from one configuration to the next. We simply assume that the initialisation equations can refer to the state prior to switching, through a construct like pre $x$, as is the case in the current Hydra implementation $[14,16]$. We leave formalisation of this, as well as a more detailed account of initialisation and reinitialisation equations to future work.

\section{Related Work}

There is a wide body of literature on the design and implementation of noncausal modelling languages. Here, we mainly focus on what is of immediate relevance to the topic of this paper: the semantics of noncausal modelling languages supporting both higher-order modelling and highly structurally dynamic systems.

To our knowledge, the first attempt to formally specify the semantics of a noncausal modelling language was Kågedal's natural semantics for a subset of Modelica [20,21]. Semantics here means 
a translation of a Modelica model into Flat Modelica: a "flat" set of equations (and a bit more: see below). Naturally, the main focus of the work was to accurately capture the meaning of Modelica's object-oriented constructs (classes, inheritance, etc.) as well as Modelica specifics like connect-equations. An additional goal of the work was the automatic generation of a Modelica implementation from the semantics. To that end, the semantics was expressed in RML, a restricted form of natural semantics that could be compiled into fairly efficient code. No attempt was made to prove any formal properties of the semantics, neither manually nor through the use of any mechanised theorem prover.

As there is no notion in Modelica of generating new structural configurations during simulation, Kågedal's semantics is entirely static: it encompasses conventional static semantics (Modelica's type system etc.) and a translation into flat equations. Modelica does have some support for hybrid systems. For example, a Modelica model can include algorithm sections that are invoked in response to events and that effect discrete changes to the model state. However, no attempt was made in Kågedal's work to capture the dynamic semantics of such constructs. Instead, after static checking, such constructs were transliterated into the output format. Thus, Flat Modelica is a rich language, including not just ordinary mathematical equations, but also an algorithmic sub language and facilities for handling events. This is in contrast to our work: the meaning of a structural configuration in our setting is given by a set of conventional mathematical equations, and our semantics does explain how the system evolves in response to events.

The standard approach for describing the semantics of hybrid systems is hybrid automata [18]. A hybrid automaton is essentially a set of states, corresponding to system configurations, along with equations that describe the dynamic behaviour of the system in each state, and when transitions between states can and must occur. In our setting, one option is thus to view a specific configuration as a fragment of a hybrid automaton, and then leverage the theory of hybrid automata to give the continuous semantics of that configuration. That would mean that our semantics can be understood as describing the construction of the reached parts of a possibly infinite hybrid automaton on demand from a high-level, declarative, system model. Taken together, the semantics presented in this paper along with the theory of hybrid automata would thus provide a complete FHM semantics.

An alternative approach to describing the continuous part of the semantics could be to follow Wan and Hudak's work on Functional Reactive Programming [29] where a discrete semantics is shown to converge to an ideal continuous semantics in the limit as the time steps approach zero. We have yet to explore this avenue in earnest. It would also be interesting to explore to what extent exact reals might be used to in a similar way.

Taking a new approach, Pepper et al. [28] give a semantics to a Modelica-style language by giving meaning directly to a modular system of components. That is, they attempt to describe the meaning of components in situ, without first flattening the system of equations. They consider an ideal semantics for continuous aspects of the language and investigate some discrete aspects relating to structural dynamism. However, unlike the work presented in this paper, they do not consider highly structurally dynamic systems, i.e., all system configurations must be computed prior to simulation, and they do so in a setting lacking first-class signal relations. They also discuss uncertainty problems related to the formulation of ideal semantics and discuss how to bring the semantics closer to the approximate solutions achieved via numerical methods. Hence, their work is in some ways complementary to our own.

Other work on formalising the semantics of higher-order modelling languages include Broman's MKL [3, 4] as well as earlier work of ours [6]. However, neither of these considered structural dynamism $^{3}$, and both give an operational semantics as opposed to the more denotational approach of this paper. Moreover, MKL is a metamodelling language: a language designed to facilitate the implementation of modelling languages (through embedding), including a type system tailored to support the metaprogramming aspects.

In contrast, Zimmer's language Sol supports both higher-order modelling and structural dynamics, but his work is centred around language design and advanced implementation techniques, only giving an informal account of the semantics [32].

Another structurally dynamic, hybrid language called Acumen is being developed by Zhu et al. [30]. However, to date, their work has been focused on automatic methods for mapping analytical models to executable code. They have focused on continuous aspects, and have yet to consider discrete aspects such as configuration changes or flattening.

Finally, there is a close correspondence between the general structure of the semantics presented here and the current Hydra implementation $[14,16]$ in that the flat sets of equations and switch conditions that result from our normalisation are exactly what gets compiled into efficient simulation code through just-in-time compilation in that implementation. Our development here was naturally partly guided by the structure of the present implementation. Consequently, our semantics suggests a feasible approach to practical implementation. Moreover, Giorgidze provides a preliminary combined discrete and continuous semantics in his dissertation [14], somewhat similar in style to that of Pepper et al.

\section{Future Work and Conclusions}

The work presented in this paper is open to numerous avenues of expansion. As already alluded to earlier in the paper, our formalisation does not account for initialisation and reinitialisation of variables across system configurations. We do not hope to provide a completely automatic solution to this problem, as consistent (re)initialisation of DAEs is well-known to be difficult in general, but we do aim to provide and formalise a core mechanism that could be used as a foundation for a number of more or less automated high-level mechanisms for state transfer.

Tying into the previous problem, one could envisage a more general mechanism for transferring data between structural configurations by allowing events to depend on a payload. In the simple case, payloads might carry instantaneous values for signal variables at the point the event was triggered, allowing for variables to be reinitialised from their values prior to the switch. In the more general case, one could allow switching blocks to be parametrised on arbitrary values, including systems of equations themselves.

Another important avenue would be to allow (co)recursively defined signal relations. As discussed earlier, one can imagine expressing a system of equations via a form of when-guarded recursion. One way to achieve this might be to adapt work by Capretta [7] on writing interpreters for languages with general recursion using the partiality monad.

A shortcoming of the current implementation is the handling of local variables. It would be preferable if local variables could be dealt with in a similar manner to the other kinds of variables, through a well-scoped relation. At present, local variable positions are simply tagged and not identified, to avoid the problem of variable clashes during normalisation. Instead one can imagine expressing as part of the model the accumulation of local variables as a term is reduced.

An orthogonal goal, the formalisation of the continuous aspects of the language could be considered, providing a complete semantics for the whole life cycle of the language without needing to

\footnotetext{
${ }^{3}$ However, MKL has recently been extended in that direction (personal communication).
} 
depend on a simulation oracle. We sketched some possible avenues in Sect. 6.

In conclusion, we have shown that $\mathrm{NbE}$ is an attractive approach for giving a denotational semantics for the discrete part of a higherorder, structurally dynamic modelling language, and we have suggested a specific structure for such a semantics with a clean interface to a separate semantics for the continuous part. Working with a fully formalised semantics in dependent type theory has already allowed us to show some key correctness aspects, such as termination of normalisation and subject reduction, and paves the way for further metatheoretical study. In particular, the work in this paper is a good starting point for further work on type systems for modelling languages; for example, for balance checking [6].

\section{Acknowledgements}

The authors would like to thank George Giorgidze and the anonymous reviewers for helpful feedback on the paper.

\section{References}

[1] U. Berger and H. Schwichtenberg. An inverse of the evaluation functional for typed lambda-calculus. In Proceedings of the Sixth Annual IEEE Symposium on Logic in Computer Science (LICS), pages 203-211, 1991.

[2] M. Boespflug. Efficient normalization by evaluation. In O. Danvy, editor, 2009 Workshop on Normalization by Evaluation, Los Angeles, USA, Aug. 2009.

[3] D. Broman. Meta-Languages and Semantics for Equation-Based Modeling and Simulation. PhD thesis, Department of Computer and Information Science, Linkping University, Sweden, 2010.

[4] D. Broman and P. Fritzson. Higher-order acausal models. In Proceedings of the 2nd International Workshop on Equation-Based ObjectOriented Languages and Tools (EOOLT), number 29 in Linköping Electronic Conference Proceedings, pages 59-69, Paphos, Cyprus, 2008. Linköping University Electronic Press.

[5] J. Capper. Agda source repository. www.cs.nott.ac.uk/ jjc.

[6] J. Capper and H. Nilsson. Static balance checking for modular systems of equations. In In Proceedings of the Eleventh Symposium on Trends in Functional Programming. Lecture Notes in Computer Science, 2010.

[7] V. Capretta. General recursion via coinductive types. Logical Methods in Computer Science, 1(2):1-18, 2005. ISSN 1860-5974. doi: 10. 2168/LMCS-1(2:1)2005. URL http://www.lmcs-online.org/ ojs/viewarticle $\cdot$ php?id=55.

[8] F. E. Cellier. Object-oriented modelling: Means for dealing with system complexity. In Proceedings of the 15th Benelux Meeting on Systems and Control, Mierlo, The Netherlands, pages 53-64, 1996.

[9] F. E. Cellier and E. Kofman. Continuous System Simulation. SpringerVerlag, 2006.

[10] T. Coquand and P. Dybjer. Intuitionistic model constructions and normalisation proofs. In Mathematical Structures in Computer Science, pages 75-94, feb 1996.

[11] N. G. de Bruijn. Lambda calculus notation with nameless dummies: A tool for automatic formula manipulation, with application to the church-rosser theorem. In Indagationes Mathematicae. Elsevier, 1972.

[12] P. Dybjer and A. Filinski. Normalisation and partial evaluation. In In Applied Semantics, Advanced Lectures. Tutorial notes from the International Summer School, Caminha, Portugal, Sept. 2000. Lecture Notes in Computer Science.

[13] C. Elliott and P. Hudak. Functional reactive animation. In Proceedings of ICFP'97: International Conference on Functional Programming, pages 163-173, June 1997.

[14] G. Giorgidze. First-class Models: On a Noncausal Language for Higher-order and Structurally Dynamic Modelling and Simulation. $\mathrm{PhD}$ thesis, School of Computer Science, University of Nottingham, Nottingham, NG8 1BB, UK, 2011.
[15] G. Giorgidze and H. Nilsson. Higher-order non-causal modelling and simulation of structurally dynamic systems. In Proceedings of the 7th International Modelica Conference, Linköping Electronic Conference Proceedings, pages 208-218, Como, Italy, Sept. 2009. Linköping University Electronic Press.

[16] G. Giorgidze and H. Nilsson. Mixed-level embedding and JIT compilation for an iteratively staged DSL. In Proceedings of the 19th Workshop on Functional and (Constraint) Logic Programming (WFLP 2010), volume 6559 of Lecture Notes in Computer Science, pages 48 65. Springer-Verlag, 2011.

[17] G. Giorgidze and H. Nilsson. Embedding a functional hybrid modelling language in Haskell. In S.-B. Scholz and O. Chitil, editors, Implementation and Application of Functional Languages: 20th International Symposium, IFL 2008, Revised Selected Papers, volume 5836 of Lecture Notes in Computer Science, pages 138-155. Springer-Verlag, 2011.

[18] T. A. Henzinger. The theory of hybrid automata. In Proceedings of the 11th Annual IEEE Symposium on Logics in Computer Science (LICS 1996), pages 278-292, 1996.

[19] P. Hudak. Modular domain specific languages and tools. In Proceedings of Fifth International Conference on Software Reuse, pages 134142, June 1998.

[20] D. Kågedal. A natural semantics specification for the equation-based modeling language Modelica. LiTH-IDA-Ex-98/48, Department of Computer and Information Science, Linköpings universitet, S-581 83, Linköping, Sweden, Oct. 1998.

[21] D. Kågedal and P. Fritzson. Generating a Modelica compiler from natural semantics specifications. In The 1998 Summer Computer Simulation Conference (SCSC'98), Reno, Nevada, U.S.A, July 1998.

[22] J. C. Mitchell and E. Moggi. Kripke-style models for typed lambda calculus. Annals of Pure and Applied Logic, 51:99-124, 1996.

[23] Modelica. Modelica - A Unified Object-Oriented Language for Physical Systems Modeling: Language Specification Version 3.2. The Modelica Association, Mar. 2010.

[24] T. A. Nils Anders Danielsson. Subtyping, declaratively: an exercise in mixed induction and coinduction. In 10th International Conference on Mathematics of Program Construction, 2010.

[25] H. Nilsson. Type-based structural analysis for modular systems of equations. Simulation News Europe, 19(1):17-28, Apr. 2009.

[26] H. Nilsson, J. Peterson, and P. Hudak. Functional hybrid modeling. In Proceedings of PADL'03: 5th International Workshop on Practical Aspects of Declarative Languages, volume 2562 of Lecture Notes in Computer Science, pages 376-390, New Orleans, Lousiana, USA, Jan. 2003. Springer-Verlag.

[27] H. Nilsson, J. Peterson, and P. Hudak. Functional hybrid modeling from an object-oriented perspective. Simulation News Europe, 17(2): 29-38, Sept. 2007.

[28] P. Pepper, A. Mehlhase, C. Höger, and L.Scholz. A compositional semantics for modelica-style variable-structure modeling. In 4th International Conference on Equation-based Object-oriented Modelling Languages and Tools, ETH Zürich, Switzerland, 2011.

[29] Z. Wan and P. Hudak. Functional reactive programming from first principles. In Proceedings of PLDI'01: Symposium on Programming Language Design and Implementation, pages 242-252, June 2000.

[30] Y. Zhu, E. Westbrook, J. Inoue, A. Chapoutot, C. Salama, M. Peralta, T. Martin, W. Taha, M. O'Malley, R. Cartwright, A. Ames, and R. Bhattacharya. Mathematical equations as executable models of mechanical systems. In ACM/IEEE First International Conference on Cyber-Physical Systems, 2010.

[31] D. Zimmer. Enhancing Modelica towards variable structure systems. In P. Fritzson, F. Cellier, and C. Nytsch-Geusen, editors, Proceedings of the 1st International Workshop on Equation-Based ObjectOriented Languages and Tools (EOOLT), number 24 in Linköping Electronic Conference Proceedings, pages 61-70, Berlin, Germany, 2007. Linköping University Electronic Press.

[32] D. Zimmer. Equation-Based Modeling of Variable-Structure Systems. $\mathrm{PhD}$ thesis, Swiss Federal Institute of Technology, Zürich, 2010. 